\title{
ANÁLISIS CINEMÁTICO DEL LANZAMIENTO DE UN FRISBEE MEDIANTE ACELERÓMETROS Y ANÁLISIS DE IMÁGENES DIGITALES
}

\author{
Daniel Alcides Mesa Restrepo, Daniel Jiménez Ramírez¹, Alejandro Restrepo Martinez². \\ 1 Estudiante de Ingeniería Mecánica, Facultad de Minas, damesar@unal.edu.co, djmenezr@unal.edu.co \\ 2 PhD en Ingeniería, Departamento de Ingeniería Mecánica Facultad de Minas, arestre5@unal.edu.co \\ 1,2 Universidad Nacional de Colombia, Medellín, Colombia.
}

\begin{abstract}
RESUMEN
En el siguiente artículo se analiza la cinemática del lanzamiento y trayectoria del frisbee utilizando la plataforma Arduino con un acelerómetro. Además, se implementa el análisis de videos digitales mediante el software cinemático Kinovea ${ }^{\circledR}$. Por medio de éstos se obtienen las aceleraciones y velocidades que toma el disco en tres diferentes tipos de lanzamientos: Backhand, forehand y elevator pass. Se halló que tanto el lanzador como el tipo de lanzamiento presentan diferencias que permiten su reconocimiento. Los resultados obtenidos muestran que el lanzador tuvo dificultades en garantizar la repetitividad en el lanzamiento backhand, con aceleraciones entre $23 \mathrm{~m} / \mathrm{s}^{2}$ y $64 \mathrm{~m} / \mathrm{s}^{2}$, lo cual mejoró en el caso del elevator pass entre $22 \mathrm{~m} / \mathrm{s}^{2}$ y $52 \mathrm{~m} / \mathrm{s}^{2}$ y en el forehand entre $50 \mathrm{~m} / \mathrm{s}^{2}$ y $73 \mathrm{~m} / \mathrm{s}^{2}$. Finalmente, se concluye como estos instrumentos describen la cinemática del lanzamiento.
\end{abstract}

Palabras clave: Arduino, cinemática, Kinovea®, lanzamiento y trayectoria del frisbee, medición aceleración.

Recibido: 15 de agosto de 2017. Aceptado: 24 de septiembre de 2018

Received: August 15th, 2017. Accepted: September 24th, 2018

\section{KINEMATIC ANALYSIS OF THE LAUNCH OF A FRISBEE USING ACCELEROMETERS AND DIGITAL IMAGE ANALYSIS.}

\begin{abstract}
In the following article analyzed the kinematics of the Frisbee launch and flight with an Arduino platform and an accelerometer. In addition, a digital video analysis was implemented using Kinovea® kinematic software. Accelerations and velocities of the disk described three types of launching: Backhand, forehand and elevator pass. We found that both the launcher and scenarios had differences that allowed their recognition. The results showed that the launcher had difficulties in ensuring repeatability in the backhand launch, with accelerations between $23 \mathrm{~m} / \mathrm{s}^{2}$ and $64 \mathrm{~m} / \mathrm{s}^{2}$. Repeatability improved in the elevator pass scenarios between $22 \mathrm{~m} / \mathrm{s}^{2}$ and $52 \mathrm{~m} / \mathrm{s}^{2}$ and in the forehand launching between $50 \mathrm{~m} / \mathrm{s}^{2}$ and $73 \mathrm{~m} / \mathrm{s}^{2}$. Finally, we conclude that these instruments describe the kinematic of the launch.
\end{abstract}

Keywords: Arduino, kinematic, Kinovea ${ }^{\circledR}$, launch and trajectory of frisbee, acceleration measurement.

Cómo citar este artículo: D. A. Mesa, D. Jiménez, A. Restrepo, "Análisis cinemático del lanzamiento de un frisbee mediante acelerómetros y análisis de imágenes digitales”, Revista Politécnica, vol. 14, no. 27 pp.9-19, 2018. https://doi.org/10.33571/rpolitec.v14n27a1 


\section{INTRODUCCIÓN}

Con el auge de la tecnología, los deportes han empezado a utilizar instrumentos para mejorar los resultados de los deportistas, sin afectar la esencia misma del deporte, obteniendo información precisa. En el contexto del ultimate existen algunos estudios que caracterizan las etapas y las variables cinemáticas asociadas al lanzamiento [1]. La anterior cita se enfoca en analizar los movimientos que debe realizar el atleta utilizando el software Kinovea 8.15. Entre los resultados, encuentran que la velocidad de salida del frisbee es de $37.2 \mathrm{~m} / \mathrm{s}$ y que el recorrido es de aproximadamente 80 metros. También se han hecho estudios para determinar parámetros cinemáticos asociados al vuelo del frisbee como el documento planteado por [2] que resalta la importancia de la sustentación, la fuerza de arrastre y el torque en el vuelo del disco. Además, el autor realiza un conjunto de mediciones de aceleración mediante un montaje electrónico fijado directamente en el disco. Entre los resultados, encuentra que para un lanzamiento normal la aceleración radial del disco es de $50 \mathrm{~m} / \mathrm{s}^{2}$ y con un recorrido de aproximadamente 4 metros. En dicho trabajo, también se realizó un montaje adicional con una cámara DSLR para analizar los datos por medio de seguimiento de imágenes mediante el software Tracker, obteniendo la posición del disco durante el lanzamiento y calculando el perfil de velocidad del vuelo.

El estudio realizado en [3] propone una simulación numérica con el método de Euler para establecer la trayectoria del vuelo del frisbee con diferentes ángulos de ataque, teniendo en cuenta las fuerzas que actúan sobre el disco. Además, establece que una velocidad común para los lanzamientos de frisbee es de $14 \mathrm{~m} / \mathrm{s}$ y para un ángulo de ataque de $5^{\circ} \mathrm{el}$ frisbee recorre, usualmente, menos de $20 \mathrm{~m}$.

En [4] se realizó una investigación con un sistema de captura de movimiento en el que se evalúa la técnica de jugadores de Disc golf de acuerdo con el nivel de experiencia durante el lanzamiento forehand. Entre los resultados se encuentra que la velocidad inicial del disco es de $27.3 \mathrm{~m} / \mathrm{s}$ y $20.2 \mathrm{~m} / \mathrm{s}$ para un jugador entrenado y no entrenado respectivamente. En [5] se realizó una investigación análoga con jugadores de ultimate, encontrando una velocidad inicial de $21.7 \mathrm{~m} / \mathrm{s}$ (recorrido $51.4 \mathrm{~m}$ ) y $20.7 \mathrm{~m} / \mathrm{s}$ (recorrido $30 \mathrm{~m}$ ) para un jugador entrenado y no entrenado respectivamente.
En [6] se realiza una revisión bibliográfica sobre los aspectos biomecánicos del lanzamiento del frisbee. El autor concluye que hay un gran número de maneras de lanzar el disco, pero muy pocas investigaciones realizadas hasta el momento. También resalta que hay un campo investigación importante en comparar el lanzamiento backhand de jugadores entrenados y no entrenados.

El presente trabajo busca identificar diferencias en tres tipos de lanzamientos del deporte ultimate, y efectuar una comparación entre los lanzamientos realizados por un jugador entrenado y no entrenado por medio de la cinemática del vuelo. Por lo que se considera la velocidad y la aceleración como parámetros cuantificables que permiten establecer diferencias.

\section{MATERIALES Y MÉTODO}

\subsection{Parámetros asociados al vuelo del frisbee}

La fuerza de sustentación ( $F L)$ es uno de los parámetros importantes que explica el vuelo del frisbee. Éste se fundamenta en el teorema propuesto por Bernoulli que plantea, de forma simplificada, que en un fluido en movimiento, la suma de la presión y la velocidad en un punto cualquiera debe permanecer constante. Esto implica que si la velocidad aumenta debe disminuir la presión. En la zona superior del frisbee, el aire tiene que atravesar una mayor distancia, por lo tanto, tiene que aumentar la velocidad y bajar la presión, mientras que en la parte inferior la distancia que recorren es menor por lo que la velocidad es baja, lo que se ve reflejado en una presión mayor [7], este gradiente de presión es lo que explica la fuerza de sustentación del frisbee.

La fuerza de arrastre (FD) es la suma de todas las fuerzas aerodinámicas que se oponen al vuelo del frisbee [7].

Por último, se encuentra que el momento angular es proporcionado por el impulso que el jugador realiza sobre el disco. El momento angular es el encargado de brindarle estabilidad al frisbee y de que pueda atravesar una mayor distancia a través de las corrientes de aire. Este es causado por la diferencia entre el centro de masa (CM) y el centro de presión (CP). El centro de masa es el punto donde el peso del frisbee se concentra y el centro de presión es donde actúan las fuerzas 
aerodinámicas [7] [8]. La distribución esquemática de las fuerzas se puede ver en la Fig.1.

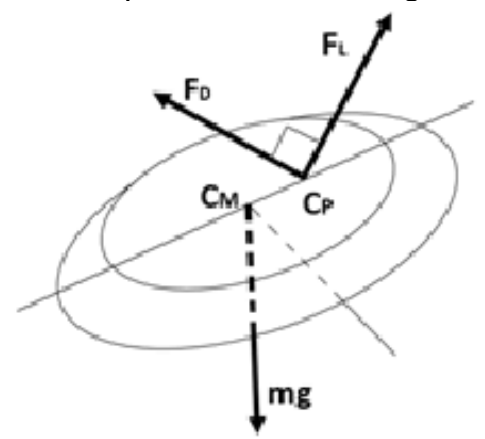

Fig.1. Fuerzas involucradas en el vuelo del frisbee. Dibujada a partir de [7].

\subsection{Consideraciones de la técnica}

La técnica tiene un papel indispensable en cualquier deporte de alto rendimiento. Ésta se define según [9] como un conjunto de procesos, que se aprenden a través del ejercicio y que se consolidan a través de la repetición.

El proceso de lanzamiento implica la acción de uno o varios movimientos. Desde la física y dinámica newtoniana [10], esto trata del desplazamiento de un objeto en el espacio, con respecto al tiempo y a un punto de referencia, y que a su vez está sometido a la inercia y fuerza.

En el deporte de ultimate existen varios lanzamientos. Para este trabajo, se analizan tres el forehand, backhand y elevator pass, debido a que presenta características cinemáticas diferenciables. Para ello es necesario entender los fundamentos básicos de cada tipo de lanzamiento:

Forehand: También conocido como el lanzamiento de dos dedos o tiro lateral, en donde el impulso viene dado de la muñeca.

Según [11], se expone tres pasos secuenciales para realizar el lanzamiento.

1. Ejecutar una rotación externa del codo y una abducción de la muñeca, para generar el momento de impulso.

2. Realizar rotación interna del codo y al tiempo la aducción de la muñeca, generando el momento de fuerza e impresión de giro.

3. Por último se procede a liberar el frisbee.
Backhand: En [11] se explica una secuencia de tres pasos para realizar este lanzamiento:

1. Ejecutar la flexión del hombro, brazo y de la muñeca seguido de una rotación interna del codo y del tronco, para generar el impulso.

2. A continuación proceder a extender el brazo, al tiempo realizar la aducción del hombro, abducción de la muñeca y la rotación del tronco en dirección contraria a la del impulso, generando el momento de fuerza.

3. Por último libera el frisbee.

Elevator Pass: Es un lanzamiento derivado del backhand y forehand, debido a que realiza el mismo movimiento del cuerpo, pero en vez de ser un pase con altura mediana se da un pequeño ángulo a la muñeca en el momento del lanzamiento, generando en la trayectoria del frisbee un efecto de lanzamiento parabólico, garantizando un pase por encima del rival [11].

\subsection{Descripción de los sistemas de medición}

\subsubsection{Sistema electrónico:}

La selección de los componentes electrónicos tuvo como principales criterios la facilidad de montaje e información disponible, los costos y la disponibilidad en el mercado. El resultado obtenido fue la elección de un Arduino UNO, un acelerómetro ADXL 335, una tarjeta MicroSD y como fuente de energía pilas CR 2032.

El dispositivo ADXL 335 es un acelerómetro que mide la aceleración en 3 ejes, el rango mínimo de escala de medición es de $\pm 3 g$ y además es un elemento de baja potencia. Está conformado por placas capacitivas fijas, placas libres y entre ellas se fijan pequeños resortes, su principio de funcionamiento radica en que cuando el movimiento se produce la distancia entre las placas fijas y libres varía, produciendo cambios de la capacitancia que se traducen en cambios de aceleración, estas señales de salida son tensiones analógicas que son proporcionales a la aceleración producida por los cambios de posición del mecanismo [12].

El Arduino UNO es el encargado de interpretar los datos que el acelerómetro envía por medio de entradas analógicas para luego almacenarlos en la tarjeta MicroSd a través de pines digitales. El lenguaje de programación del Arduino está basado en $\mathrm{C}++$ y fue diseñado con las relaciones para que los cambios de capacitancia se tradujeran 
directamente en cambios de aceleración. La frecuencia de la toma de datos, para este caso es de un dato cada 50 milisegundos con la finalidad de obtener tendencias de aceleración más precisas. Es importante mencionar que para almacenar los datos en la tarjeta MicroSd es necesario que se encuentre en formato FAT 16 o FAT 32, ya que son las librerías que actualmente soporta el sistema de Arduino.

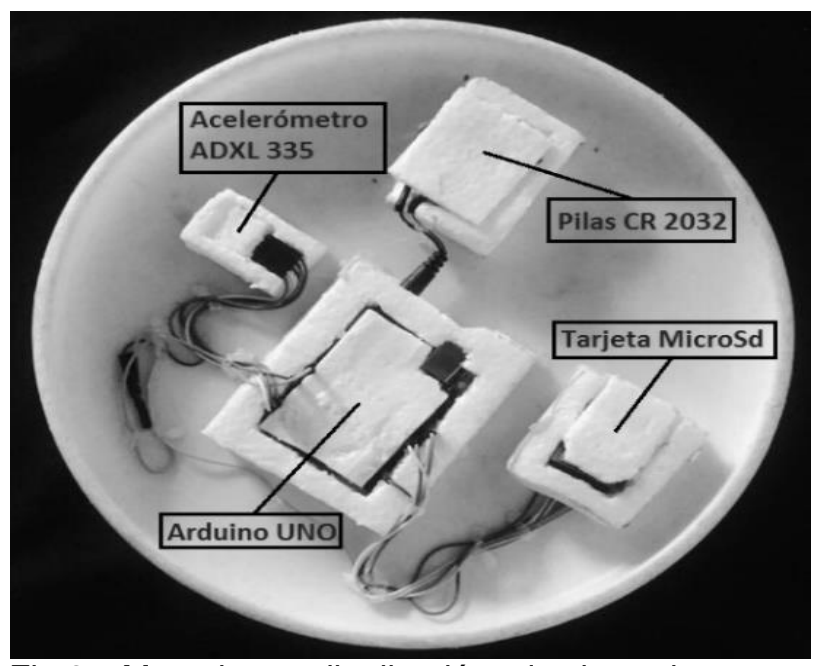

Fig.2. Montaje y distribución de los elementos electrónicos en el frisbee.

Tabla 1. Parámetros generales del montaje

\begin{tabular}{|c|c|}
\hline Parámetro & Magnitud \\
\hline Peso frisbee & $254 \mathrm{~g}$ \\
\hline Peso del montaje & 76,6 \\
\hline Diámetro frisbee & $25 \mathrm{~cm}$ \\
\hline
\end{tabular}

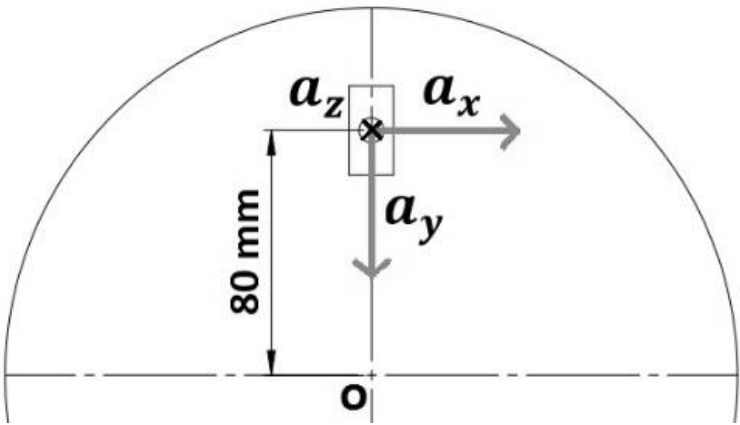

Fig.3. Dirección de las componentes de aceleración en el montaje.

Al momento de distribuir los elementos del sistema electrónico en el disco se debe garantizar que el centro de masa no se modifique, y que no afecte el agarre, ni la atrapada del disco y que además se optimice al máximo el peso, En la Fig.2 se puede ver la distribución de los elementos en el disco y en la tabla 1 se encuentran el peso final que se obtuvo con el montaje y corresponde a menos de $1 / 3$ del peso del frisbee.

El código implementado en el Arduino permitió obtener directamente la aceleración en los tres ejes coordenados. La orientación de cada aceleración y la ubicación del acelerómetro se pueden evidenciar en la Fig.3 A partir de estas variables se puede obtener la magnitud de la aceleración total como se muestra en la ecuación 1.

$$
a_{\text {Total }}=\sqrt{\left(a_{x}\right)^{2}+\left(a_{y}\right)^{2}+\left(a_{z}\right)^{2}} \quad\left[\frac{m}{s^{2}}\right]
$$

\subsubsection{Software de análisis digital:}

En la actualidad se implementan programas de análisis digital para mejorar el entrenamiento de atletas, con la intensión de mejorar su rendimiento en el deporte que practiquen y los métodos de recuperación. Estos programas permiten analizar el movimiento de los deportistas por medio de videos, en donde se aprecia el desplazamiento del atleta en una situación en específica. Algunos de los programas que tienen esta finalidad son: Hudl Technique, Coach's Eye, Track Eye y Kinovea ${ }^{\circledR}$ 0.8.15.

La implementación de Kinovea $\AA 0.8 .15$ en este proyecto, se debe a que es un software de libre uso y una herramienta de fácil interacción, que permite obtener a través del video: la posición del elemento en un instante de la grabación, el tiempo y la trayectoria del cuerpo. Los datos de coordenadas y de tiempo obtenidos por el programa, se emplean en obtener velocidades del frisbee en todo su trayecto.

En la Fig.4 se muestra el desplazamiento del frisbee (línea continua gruesa), el tiempo en cada posición en milisegundos y la ubicación del disco dentro del plano en píxeles; siendo el primer valor la posición en $\mathrm{X}$ y el segundo la posición en $\mathrm{Y}$. El video se obtuvo por medio de un celular Samsung J5 que está compuesto por una cámara de 13 megapíxeles con capacidad de grabación de 1080p a $60 \mathrm{fps}$, se opta por esta cámara ya que es un estudio académico que tiene como finalidad ser replicado por cualquier persona que incursione en estudios similares. 


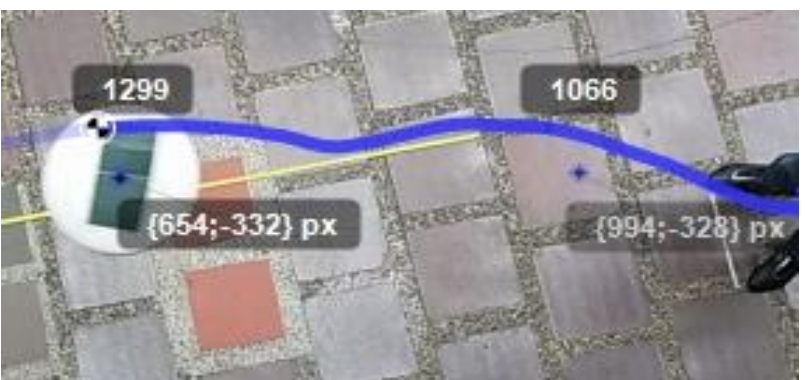

Fig.4. Trayectoria del frisbee, empleando el software Kinovea ${ }^{\circledR}$ 0.8.15.

La aplicación usa estas unidades para aproximar una medida de velocidad. Cada vez que el programa cambia de imagen toma la posición del objeto a través de los píxeles y realiza una resta de la ubicación actual con la anterior, obteniendo una diferencia en el desplazamiento del elemento, y con la diferencia de tiempo que hay entre imágenes se obtiene una velocidad en cada componente $(X, Y)$. Usando la ecuación de velocidad media, el programa muestra una velocidad del objeto en todo el trayecto.

Aplicando la ecuación 2 de velocidad media total

$$
\bar{v}_{T}=\sqrt{\left(\frac{\Delta x}{\Delta t}\right)^{2}+\left(\frac{\Delta y}{\Delta t}\right)^{2}}\left[\frac{m}{s}\right]
$$

Donde $\quad \bar{v}_{T}$ es la velocidad media total, $\Delta \mathrm{x}$ es la diferencia de desplazamiento en el eje $X, \Delta y$ es la diferencia de desplazamiento en el eje $Y$ y $\Delta$ t es la diferencia de tiempo entre la imágenes.

\section{RESULTADOS Y DISCUSIÓN}

\subsection{Resultados del sistema electrónico}

En general, se encontraron comportamientos similares en las gráficas de aceleración total de los lanzamientos backhand y forehand. En la Fig.5 se muestra un lanzamiento específico que ejemplifica estos comportamientos. Analizando e interpretando los videos de los lanzamientos y comparándolos con las gráficas obtenidas con el montaje electrónico, se encontró que entre el punto 1-2 el lanzador lleva el frisbee hacia atrás para empezar el lanzamiento. Luego entre 2-3 el jugador impulsa el disco hacia adelante hasta llegar al punto 3 que corresponde a la aceleración máxima que el jugador alcanza cuando realiza la aducción de la muñeca, generando la rotación del frisbee, a partir de este punto la aceleración decrece hasta el punto 4 debido a que el lanzador no suelta inmediatamente el disco. Entre 4-5 está comprendido el vuelo del disco cuya extensión y comportamiento depende fuertemente del tipo de lanzamiento. Entre 5-6 se presenta un pico de aceleración que es causado por el impacto y la reacción del jugador para atrapar el disco y a partir de 6 se desacelera completamente el frisbee.

El comportamiento de la aceleración del lanzamiento elevator pass difiere de los demás. En la Fig.6 se muestra la gráfica de un lanzamiento específico que ejemplifica el comportamiento general. La primera diferencia identificada, es que en la técnica no se necesita generar un impulso relevante hacia atrás, debido a que la distancia para este lanzamiento es muy corta, por lo que la figura no presenta un descenso en la aceleración. Entre 1-2 se genera el impulso del frisbee hacia adelante, a partir del punto 2 el jugador desacelera el movimiento, con la finalidad de que entre 3-4 el jugador logre realizar la aducción de muñeca y al mismo tiempo realizar el movimiento para que el frisbee ascienda. Entre 4-5 en esta comprende el vuelo del disco. En el punto 5 se ejecuta la atrapada del disco y al igual que en la Fig. 5 se puede dar un pico que dependerá de la atrapada. Finalmente, entre 5-6 se desacelera el frisbee.

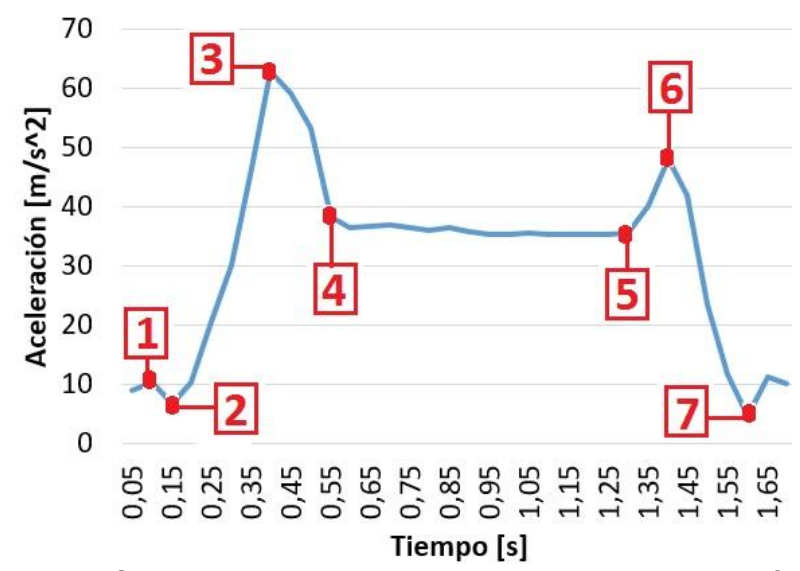

Fig.5. Comportamiento general de la aceleración total de los lanzamientos backhand y forehand.

Para evaluar la capacidad de un jugador para ejecutar un conjunto de lanzamientos de un mismo tipo garantizando la repetitividad, es necesario determinar qué tan hábil es el lanzador para realizar movimientos precisos. Para ello, se analizan cambios en la aceleración de un conjunto de lanzamientos, evaluando similitudes y diferencias. La distancia entre el lanzador y el receptor es de 4 metros. 


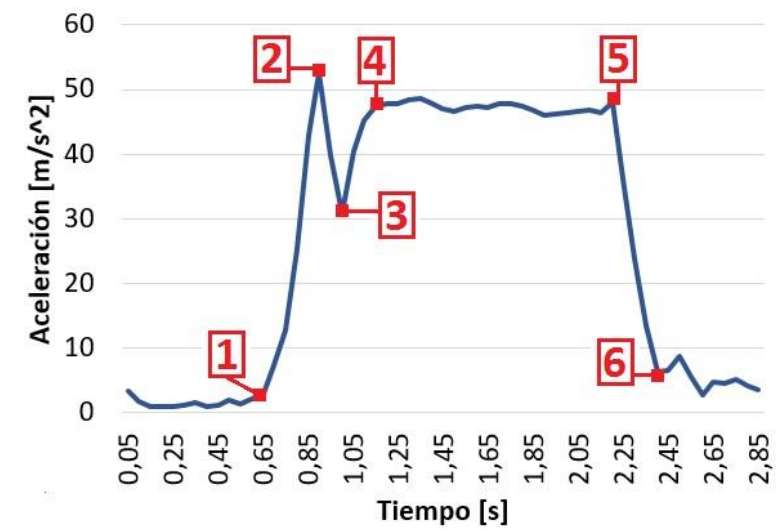

Fig.6. Comportamiento general de la aceleración total del lanzamiento elevator pass.

Analizando las Fig.7, Fig.8 y Fig.9, se observa que en general la tendencia es similar en cada una, pero se encuentran diferencias en los tiempos de ejecución de la técnica y en la permanencia del frisbee en vuelo. En la tabla 2 se muestran los tiempos y aceleraciones más relevantes. Estos fueron obtenidos promediando los 14 lanzamientos de cada tipo de lanzamiento, siendo backhand (B), forehand $(\mathrm{F})$ y elevator pass $(\mathrm{EP})$.

Tabla 2. Aceleraciones y tiempos promedios en la etapa de lanzamiento y vuelo del frisbee.

\begin{tabular}{|l|c|c|c|}
\hline \multicolumn{1}{|c|}{ Parámetro } & B & F & EP \\
\hline $\begin{array}{l}\text { Tiempo de ejecución de } \\
\text { la técnica [s] }\end{array}$ & 0,45 & 0,45 & 0,55 \\
\hline $\begin{array}{l}\text { Tiempo de vuelo del } \\
\text { frisbee [s] }\end{array}$ & 0,65 & 0,55 & 1,05 \\
\hline $\begin{array}{l}\text { Aceleración máxima al } \\
\text { impulsar el frisbee [m/s }\end{array}$ & 76 & 81 & 56 \\
\hline $\begin{array}{l}\text { Aceleración mínima al } \\
\text { impulsar el frisbee [m/s }\left[\mathrm{s}^{2}\right]\end{array}$ & 54 & 49 & 44 \\
\hline $\begin{array}{l}\text { Aceleración máxima del } \\
\text { vuelo del frisbee }\left[\mathrm{m} / \mathrm{s}^{2}\right]\end{array}$ & 64 & 73 & 52 \\
\hline $\begin{array}{l}\text { Aceleración mínima del } \\
\text { vuelo del frisbee }\left[\mathrm{m} / \mathrm{s}^{2}\right]\end{array}$ & 23 & 50 & 22 \\
\hline
\end{tabular}

En el análisis se evidencia que los lanzamientos backhand y forehand tienen tiempos similares, mientras que el elevator pass tiene notables diferencias. En cuanto al tiempo de ejecución de la técnica no hay diferencias significativas en los tres lanzamientos, sin embargo, es importante resaltar que el lanzamiento elevator pass necesita un tiempo más corto para realizar el impuso del frisbee hacia adelante, pero un tiempo más prolongado para imprimirle el efecto ascendente al disco, ya que según el video, el jugador realiza un movimiento adicional que implica desacelerar y acelerar el disco en este intervalo de tiempo, lo que al mismo tiempo causa que la aceleración máxima de vuelo esté por debajo de los otros lanzamientos. En el lanzamiento elevator pass, el tiempo de vuelo del frisbee más elevados que los demás lanzamientos, el disco recorre una mayor distancia a una aceleración menor (ver tabla 2). Mientras que en los otros lanzamientos hay leves diferencias, esto se debe a que en la técnica del forehand requiere una mayor aceleración que el backhand, para generar una adecuada estabilidad y efecto al disco.

Para contrastar los resultados de los lanzamientos de un jugador inexperto con un jugador entrenado, se realizaron varios ensayos con las mismas restricciones. El comportamiento de los lanzamientos backhand mostrados en la Fig.10, tienen diferencias en los intervalos de tiempo como en aceleraciones, respecto a un jugador entrenado. En la tabla 3, se pueden identificar los parámetros más relevantes de los resultados.

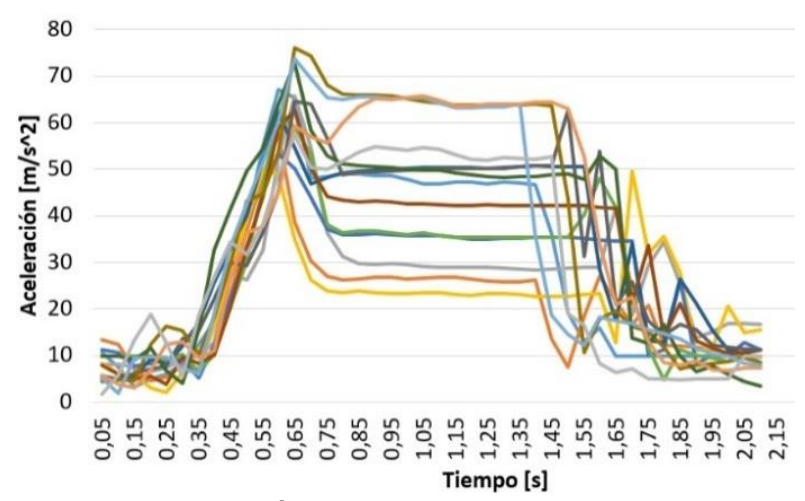

Fig.7. Aceleración total de los lanzamientos backhand realizado por un jugador entrenado. Total de lanzamientos: 14.

En primera instancia, los intervalos de tiempo promedio identificados en la gráfica son similares, sin embargo, los datos obtenidos del lanzador inexperto presentan una mayor dispersión en los intervalos de tiempos del vuelo, por lo que la repetitividad del lanzamiento en este aspecto es variable. En segunda instancia, el intervalo de aceleración para impulsar el frisbee es el mismo, sin embargo, el rango de aceleraciones del jugador no entrenado es menor, esto indica que, en promedio, el jugador no entrenado imprime menos impulso al momento de realizar el lanzamiento. Además, el tiempo de vuelo del disco es menor, 
esto se fundamenta en que el jugador inexperto, en general, desacelera el frisbee en una menor proporción para realizar el lanzamiento.

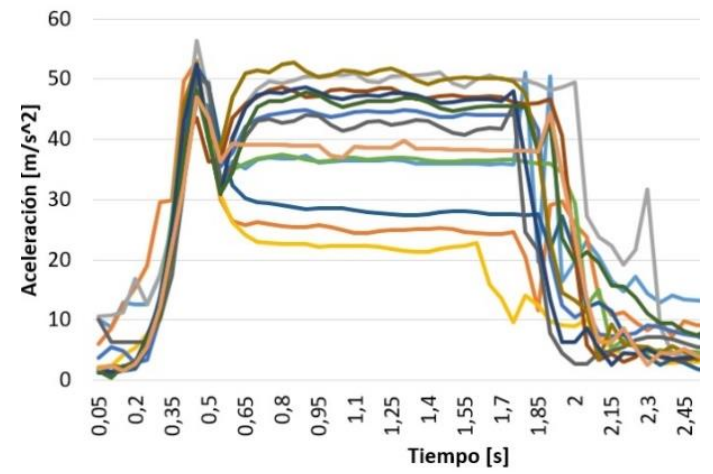

Fig.8, Aceleración total de los lanzamientos elevator pass realizado por un jugador entrenado. Total de lanzamientos: 14.

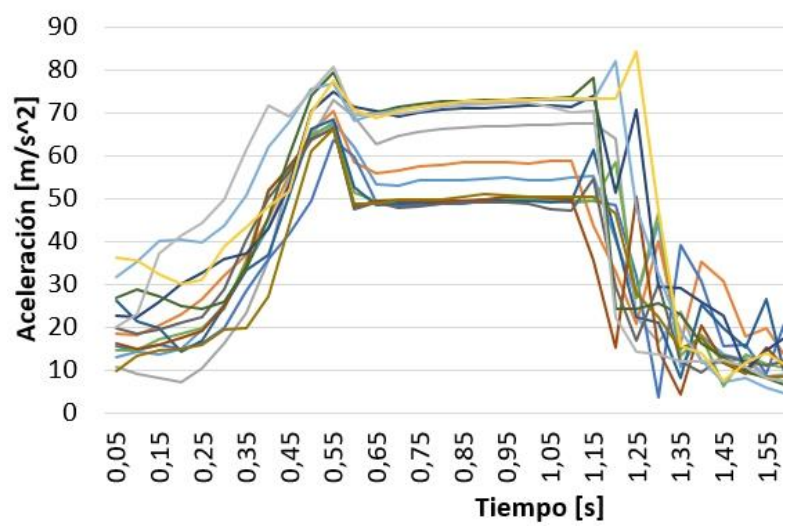

Fig.9. Aceleración total de los lanzamientos forehand realizado por un jugador entrenado. Total de lanzamientos: 14.

Tabla 3. Comparación de parámetros de los lanzamientos backhand realizados por un jugador entrenado (B. E.) y un jugador no entrenado (B. I.).

\begin{tabular}{|l|c|c|}
\hline \multicolumn{1}{|c|}{ Parámetro } & B.E & B.I \\
\hline Tiempo de ejecución de técnica [s] & 0,45 & 0,5 \\
\hline Tiempo de vuelo del frisbee [s] & 0,65 & 0,6 \\
\hline $\begin{array}{l}\text { Aceleración máxima al impulsar el } \\
\text { frisbee [m/s²] }\end{array}$ & 76 & 53 \\
\hline $\begin{array}{l}\text { Aceleración mínima al impulsar el } \\
\left.\text { frisbee [m/s } \mathrm{s}^{2}\right]\end{array}$ & 54 & 31 \\
\hline $\begin{array}{l}\text { Aceleración máxima del vuelo del } \\
\text { frisbee [m/s } \mathrm{s}^{2} \text { ] }\end{array}$ & 64 & 45 \\
\hline $\begin{array}{l}\text { Aceleración mínima del vuelo del } \\
\text { frisbee }\left[\mathrm{m} / \mathrm{s}^{2}\right]\end{array}$ & 26 & 27 \\
\hline
\end{tabular}

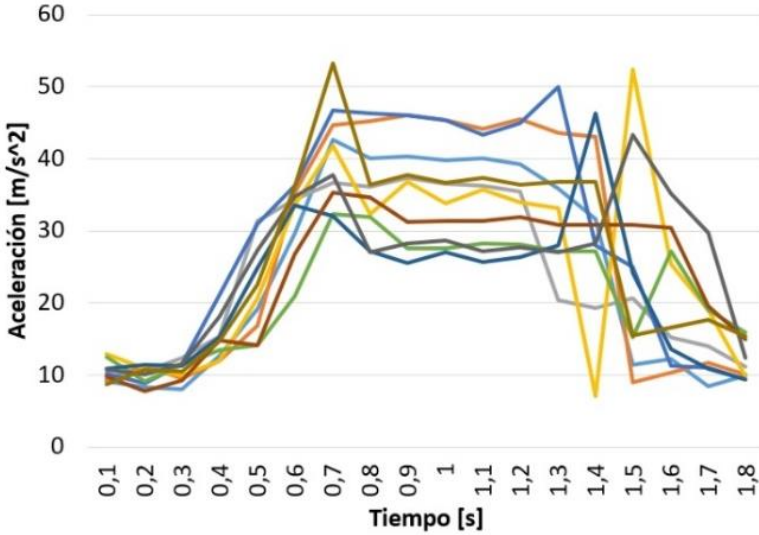

Fig.10. Aceleración total de los lanzamientos backhand realizados por un jugador no entrenado. Total de lanzamientos: 10.

En el lanzamiento elevator pass realizado por un jugador no entrenado (ver Fig.11), tiene diferencias apreciables en los diferentes intervalos de tiempo y aceleración expuestos en la tabla 4.

En primer lugar, el tiempo de ejecución de la técnica es menor. Esto indica, según los videos, que el jugador inexperto no realiza un paso, que visto desde el comportamiento de las gráficas, se manifiesta en desacelerar el frisbee y luego nuevamente impulsarlo. Esto se refleja en que la trayectoria parabólica que realiza el disco no es tan pronunciada comparada con la realizada por el jugador entrenado. La omisión del paso tiene incidencia directa en el tiempo de vuelo del frisbee, ya que disminuye el rango de aceleraciones en el desplazamiento del disco en el aire.

Tabla 4. Comparación de los parámetros de los lanzamientos elevator pass realizados por un jugador entrenado (B. E.) y un jugador no entrenado (B. I.)

\begin{tabular}{|l|c|c|}
\hline \multicolumn{1}{|c|}{ Parámetro } & B.E & B.I \\
\hline Tiempo de ejecución de técnica [s] & 0,55 & 0,5 \\
\hline Tiempo de vuelo del frisbee [s] & 1,05 & 1,3 \\
\hline $\begin{array}{l}\text { Aceleración máxima al impulsar el } \\
\text { frisbee [m/s }{ }^{2} \text { ] }\end{array}$ & 56 & 65 \\
\hline $\begin{array}{l}\text { Aceleración mínima al impulsar el } \\
\left.\text { frisbee [m/s } \mathrm{s}^{2}\right]\end{array}$ & 44 & 31 \\
\hline $\begin{array}{l}\text { Aceleración máxima del vuelo del } \\
\text { frisbee [m/s } \mathrm{s}^{2} \text { ] }\end{array}$ & 52 & 48 \\
\hline $\begin{array}{l}\text { Aceleración mínima del vuelo del } \\
\text { frisbee }\left[\mathrm{m} / \mathrm{s}^{2} \text { ] }\right.\end{array}$ & 22 & 11 \\
\hline
\end{tabular}




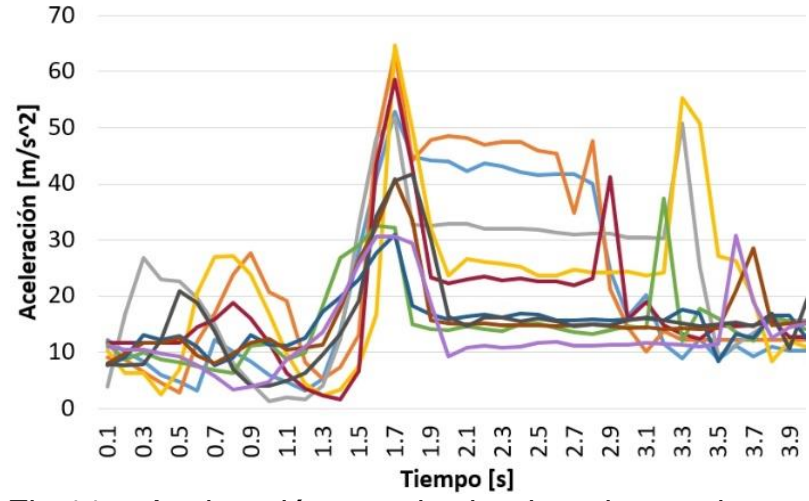

Fig.11. Aceleración total de los lanzamientos elevator pass realizado por un jugador no entrenado. Total de lanzamientos: 10.

El comportamiento de las componentes de aceleración presenta variaciones importantes en los lanzamientos backhand (ver Fig.13) y elevator pass (ver Fig.12). En la tabla 5, se muestran las magnitudes de las aceleraciones más relevantes de un lanzamiento específico que ejemplifica el comportamiento general. En primer lugar se destaca, que en el elevator pass la componente de la aceleración en el eje vertical (Az) tiene una magnitud considerable en el vuelo del frisbee, inclusive similar a la aceleración (Ay) del disco.

Tabla 5. Comparación de las componentes de aceleración promedio en la etapa de vuelo del lanzamiento elevator pass (EP) y backhand (B).

\begin{tabular}{|l|c|c|}
\hline Aceleración del vuelo del disco & EP & B \\
\hline Aceleración en el eje $X\left[\mathrm{~m} / \mathrm{s}^{2}\right]$ & -1 & -1 \\
\hline Aceleración en el eje $\mathrm{Y}\left[\mathrm{m} / \mathrm{s}^{2}\right]$ & 27 & 36 \\
\hline Aceleración en el eje Z $\left[\mathrm{m} / \mathrm{s}^{2}\right]$ & 25 & -1 \\
\hline Aceleración total $\left[\mathrm{m} / \mathrm{s}^{2}\right]$ & 37 & 36 \\
\hline
\end{tabular}

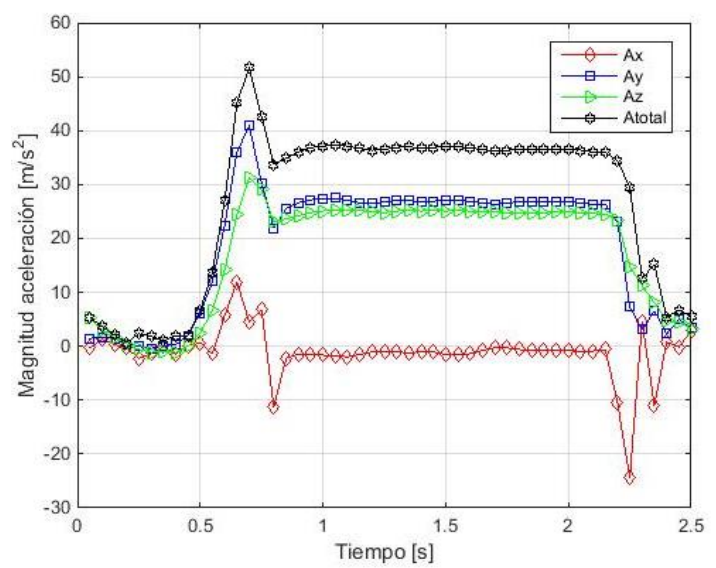

Fig.12. Componentes de la aceleración y aceleración total de un lanzamiento elevator pass.

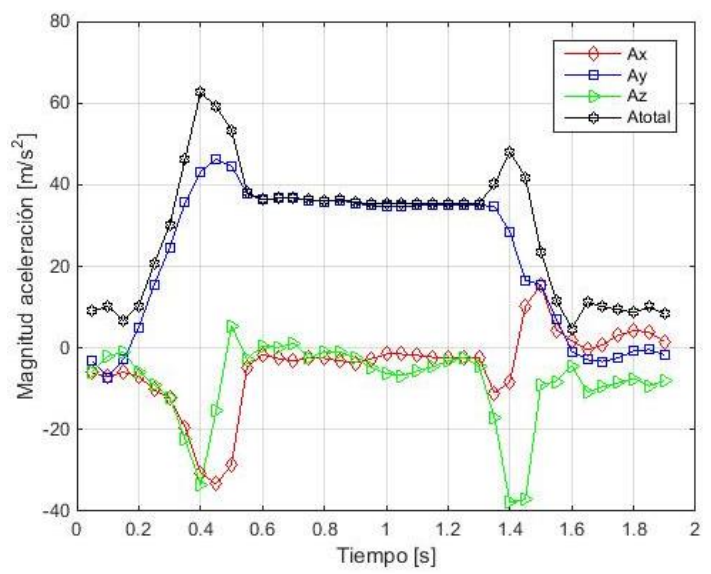

Fig.13. Componentes de la aceleración y aceleración total de un lanzamiento backhand.

Este comportamiento de las aceleraciones da cuenta de la incidencia de la aceleración vertical en el movimiento parabólico del frisbee, por lo que despreciarla en el análisis de video aporta un error importante en los resultados. Por otra parte, en el lanzamiento backhand la componente de aceleración vertical sólo cobra importancia en la etapa de lanzamiento, lo que indica que el jugador en la ejecución del lanzamiento impulsa verticalmente el frisbee y luego lo lanza en una trayectoria sin mayores variaciones verticales. Lo anterior aporta errores menores en las simplificaciones del análisis por imágenes digitales. La componente de la aceleración en el eje coordenado $\mathrm{X}(\mathrm{Ax})$ es cercano a cero en ambos lanzamientos durante la etapa de vuelo (ver tabla 5 ), esto se debe a que el disco tiene velocidad tangencial constante. Esto implica que el frisbee al volar presenta características similares a un movimiento circular uniforme.

\subsection{Resultados del análisis de imagen}

Por medio del análisis de imagen, se obtienen las siguientes gráficas para cada tipo de lanzamiento, en las que se aprecian las divisiones de zonas. La zona 1 comprende el transcurso de tiempo en el cual el lanzador está generando el momento de fuerza para liberar el disco, en la zona 2 se presenta la velocidad total del vuelo del frisbee y por último en la zona 3 se muestra la velocidad total en el transcurso de tiempo cuando es atrapado el disco. La distancia entre el lanzador y el receptor es de 4 metros. 
En la Fig.14 se observa que la velocidad total promedio a la cual vuela el frisbee, alcanzada por el jugador entrenado para el lanzamiento backhand es $18 \mathrm{~m} / \mathrm{s}$.

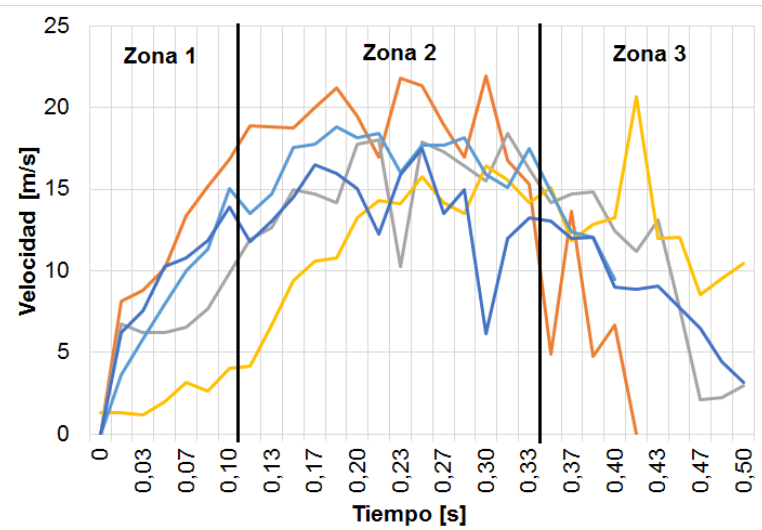

Fig.14. Velocidad total de los lanzamientos backhand realiza por un jugador entrenado. Total de lanzamientos: 5.

En la Fig.15 se muestra que la velocidad total promedio a la cual vuela el frisbee, alcanzada por el jugador entrenado para el lanzamiento forehand es 20 m/s. En la Fig.16, se aprecia que la velocidad promedio a la cual vuela el frisbee alcanzada en el lanzamiento elevator pass es $12 \mathrm{~m} / \mathrm{s}$.

Además, en la Fig.16 las curvas presentan un crecimiento y decrecimiento, con más frecuencia que en las anteriores curvas de los lanzamientos backhand y forehand, esto se debe a que el lanzamiento elevator pass tiene su mayor cambio en el eje z, y el software no puede evaluar esta variable.

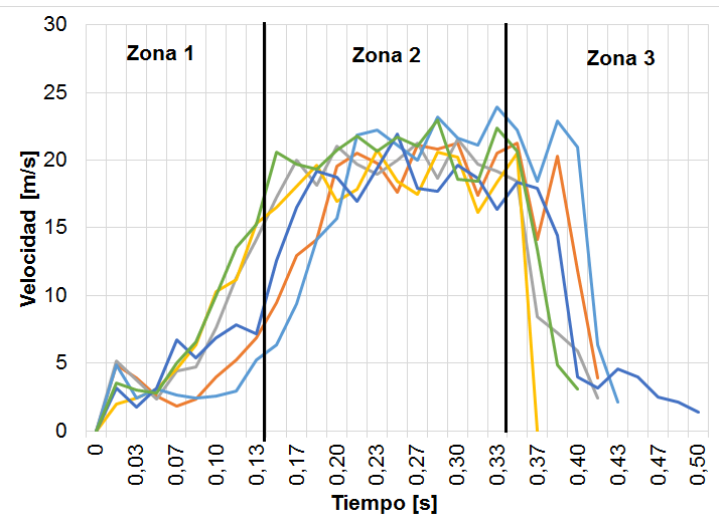

Fig.15. Velocidad total de los lanzamientos forehand realiza por un jugador entrenado. Total de lanzamientos: 6.

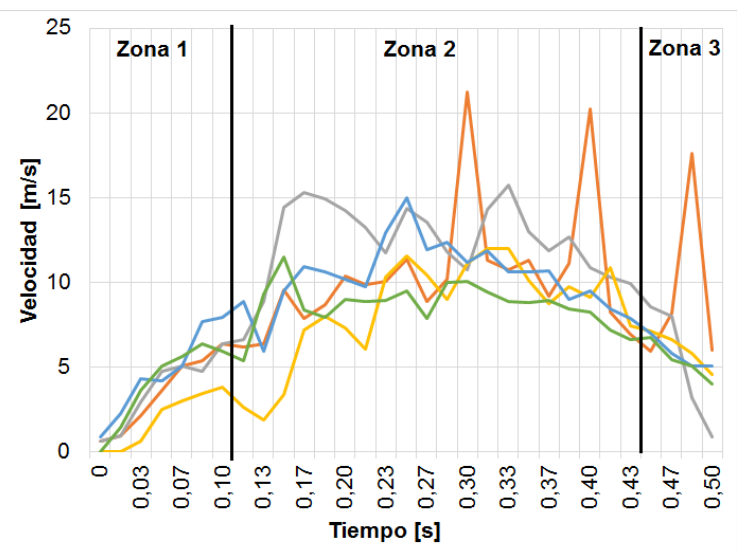

Fig.16. Velocidad total de los lanzamientos elevator pass realiza por un jugador entrenado. Total de lanzamientos: 5.

En la Fig.17 se presentan los lanzamientos realizados por un jugador no entrenado para el caso del lanzamiento backhand. Analizando la trayectoria del disco en todo el proceso se puede observar que estas curvas son más suaves que las del lanzamiento del jugador entrenado, esto es debido a que el jugador inexperto le proporciona menos aceleración al frisbee generando una menor velocidad en su trayecto, una velocidad total promedio de $6 \mathrm{~m} / \mathrm{s}$ en la zona 2, permitiendo garantizar que el programa Kinovea ${ }^{\circledR} 0.8 .15$ logre captar una referencia en el elemento, con la cual se obtiene valores de posición con menor distancia entre ellos.

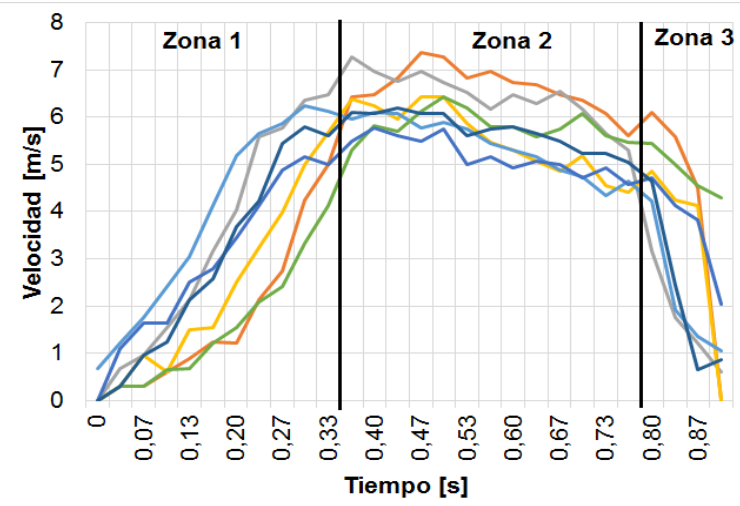

Fig.17. Velocidad total de los lanzamientos backhand realiza por un jugador no entrenado. Total de lanzamientos: 7. 


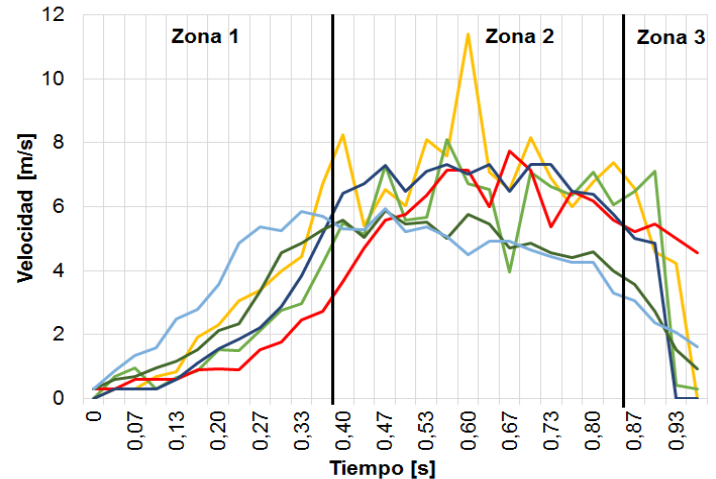

Fig.18. Velocidad total de los lanzamientos forehand realiza por un jugador no entrenado. Total de lanzamientos: 6.

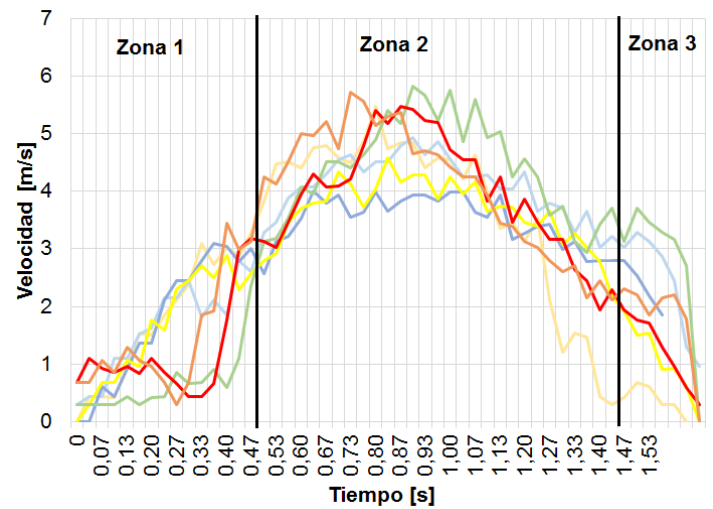

Fig.19. Velocidad total de los lanzamientos elevator pass realiza por un jugador no entrenado. Total de lanzamientos: 7.

Además, se observa que las velocidades totales de un jugador no entrenado tienen una magnitud menor que las de un jugador entrenado. La velocidad total aproximada de un jugador inexperto en la zona 2 para los lanzamientos de forehand y elevator pass van de 5 a $8 \mathrm{~m} / \mathrm{s}$ y de 4 a $6 \mathrm{~m} / \mathrm{s}$ respectivamente (ver Fig.18 y Fig.19). En un jugador entrenado los valores de velocidad totales alcanzados aproximadamente son de 16 a $25 \mathrm{~m} / \mathrm{s}$ y de 6 a $14 \mathrm{~m} / \mathrm{s}$ en los lanzamientos de forehand y elevator pass respectivamente.

En las gráficas presentadas se observan algunos picos, debido a que el programa Kinovea $\AA^{\circledR}$ 0.8.15, no logra seguir una referencia en el objeto de análisis en todo su trayecto, por lo tanto los valores obtenidos como resultado del análisis de movimiento dan valores $\Delta \mathrm{x}$ grandes para un mismo
$\Delta t$, consiguiendo como resultado una velocidad de magnitud grande (ver Fig.4). La trayectoria y la referencia dentro del plano de Kinovea $\AA^{\circledR} 0.8 .15$ se ven afectadas por condiciones externos dentro del video como son los contrastes de la iluminación y los colores, también se puede ver perturbados debido los frames por segundos de la cámara.

Los resultados obtenidos con Kinovea $\AA^{\circledR} 0.8 .15$ son acertados debido a que el contraste del marcado es apropiado con respecto al fondo (ver Fig.4), por lo tanto, no se requiere un fondo completamente modificado.

Se recomienda el uso de un fondo y un frisbee con alto contraste, como se observa en la Fig. 20, esto con el fin de evitar ruidos y picos en la curva.

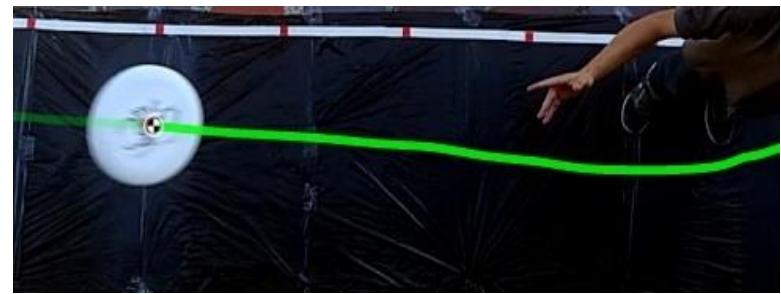

Fig. 20. Trayectoria del frisbee con ambiente controlado.

\section{CONCLUSIONES}

Mediante la implementación de los dos métodos de medición se lograron obtener resultados diferenciables en los tres tipos de lanzamientos. En donde se identificó que el comportamiento de vuelo de los lanzamientos backhand y forehand son similares, mientras que estos difieren del elevator pass. Esta diferencia se manifiesta en la magnitud de la aceleración, magnitud de la velocidad, los tiempos de ejecución de la técnica y el tiempo de permanencia del frisbee en vuelo.

El jugador entrenado logra lanzar con más facilidad que el jugador inexperto, esto se debe a que tienen una repetitividad en los lanzamientos, con una menor variabilidad en las aceleraciones y velocidades para las condiciones propuestas. Lo anterior indica que tienen una técnica de lanzamiento aprendida. Además, se identifica la misma tendencia reportada por [4] y [5], en la que un jugador entrenado lanza con mayor velocidad que un jugador no entrenado. 
Los valores obtenidos de velocidad promedio para un jugador entrenado en el lanzamiento forehand $(20 \mathrm{~m} / \mathrm{s})$ son similares a los valores reportados por [4] para el mismo lanzamiento $(27.3 \mathrm{~m} / \mathrm{s})$. Lo que indica que así no se haya tenido un ambiente completamente controlado, los resultados finales se encuentran entre los rangos establecidos en la literatura. Sin embargo, se recomienda realizar las pruebas en un ambiente controlado, utilizar una cámara con mayor frames por segundo, un circuito integrado para disminuir el peso y un acelerómetro con giroscopio (esté con el fin de evaluar el comportamiento rotacional del disco).

Los entrenadores por medio de los métodos expuestos en este informe podrán encontrar falencias en la técnica de los deportistas, utilizando análisis de datos y otras fuentes similares al tema, con el fin de encontrar nuevas herramientas que permitan la evaluación de la técnica.

\section{AGRADECIMIENTOS}

Los autores agradecen al compañero Jhon Pérez; jugador del equipo de ultimate de la Universidad Nacional de Colombia sede Medellín, por su colaboración en las pruebas realizadas.

\section{REFERENCIAS BIBLIOGRÁFICAS}

[1] Jáuregui, J. A., \& Tejada Otero, C. P. "Análisis biomecánico del lanzamiento pull-backhand en Ultimate Frisbee". Colombia. VIREF Biblioteca Virtual de Educación Física, Vol.3, 2014.

[2] J. Leeman, "The flight of the frisbee", (2015, 24 May). [Online]. Available: http://www.johnrleeman.com/2015/05/24/the-flightof-the-frisbee/.

[3] V.R.Morrison, "The Physics of Frisbees", Physics Department, Mount Allison University, Electronic Journal of classical Mechanics and Relativity, Mount Allison University Sackville, NB Canada E4L 1E6, 2005.

[4] H. Jung, S. Kim, M. Choi, J. Lee and K. Lee, "A qualitative comparison of disc movement and inter joint coordination of upper limb in flying disc forehand throwing." kookmin University.

[5] K. Sasakawa and S. Sakurai, "Biomechanical analysis of the sidearm throwing motion for distance of a flying disc: A comparison of skilled and unskilled Ultimate players", Sports Biomechanics, vol. 7, no. 3, 311-321, 2008.

[6] B. Schultze, "Biomechanical aspects of throwing a frisbee: A review" [Bachelor Thesis], Münster, Münster University, 2016.

[7] S. Hummel, "Frisbee Flight Simulation and Throw Biomechanics", Master of Science in Mechanical Engineering, University of California, 2003.

[8] A. Valderrama and J. Valderrama, "Trabajo Semestral Guiado: un Buen Complemento al Estudio y Aprendizaje en Cursos de Ciencia y de Ingeniería", Formación universitaria, vol. 7, no. 3, pp. 3-10, 2014.

[9] R. Manno, Fundamentos del entrenamiento deportivo, 1st ed. Barcelona: Paidotribo, 1994.

[10] R. Gomez, "Del movimiento a la acción motriz: elementos para una genealogía de la motricidad", Educación Física y Ciencia, vol. 14, pp. 49-50, 2012.

[11] C. Tejada Otero, "Ultimate Frisbee Metodología de Entrenamiento", 1st ed. Colombia: VIREF Biblioteca Virtual de Educación Física, 2009.

[12] Analog Device, Inc, Datasheet "Accelerometer ADXL 335.", USA, 2009. 